\title{
An International Comparative Study of the Connotations of Social Responsibility
}

\author{
Su Lana, XiongTing ${ }^{b}$ \\ 1Jiangxi Normal University Nanchang 330022 \\ 2Jiangxi Open University Nanchang 330046 \\ a20227879@qq.comb23477082@qq.com
}

Corresponding author: Su Lan, Jiangxi normal university, no.99, ziyang road, nanchang, jiangxi province, China 330022

Keywords: social responsibility, connotations, international comparison

\begin{abstract}
Social responsibility is a moral indicator of national modernization and social civilization. It is also an integral part of individual modernization and an important guarantee for social progress. For the connotations of social responsibility, domestic research focuses on three perspectives, including ethics and morals, social life and corporate development, while overseas research on this subject revolve around three theories, namely on goals, factors and management. A rational perception of the connotations of social responsibility and an in-depth analysis of the dual dimension of ethics and governance contained in social responsibility are conducive to more profound, systematic research in the future.
\end{abstract}

\section{社会责任品质内涵的国际比较研究}

\author{
苏兰 ${ }^{a}$, 熊婷 ${ }^{b}$ \\ 1 江西师范大学, 南昌, 江西, 中国 \\ 2 江西广播电视大学, 南昌, 江西, 中国 \\ a20227879@qq.com, b23477082@qq.com
}

通讯作者:中国江西省南昌紫阳大道 99 号江西师范大学苏兰收 330022

关键词: 社会责任品质; 内涵; 国际比较

摘要: 社会责任品质作为标志国家现代化水平和社会文明程度的道德品质, 是个体现代化发展的重要 内容, 也是社会发展的重要保障。国内关于社会责任品质内涵的研究主要从伦理道德视角、社会生活 视角和企业发展视角三方面进行阐释; 国外关于社会责任品质内涵的研究主要包括目标论、要素论和 管理论三种观点。理性认知社会责任品质的内涵, 透析社会责任品质所包含的伦理和治理的双重维度, 有利于未来更加深入、系统的研究。

\section{1. 引言}

在人类社会历史领域，人的社会责任是绝对的。个人必须对自己的一切负责，既要对决 定他的社会环境负责，也要对影响他自我负责的一切社会条件负责。[1]“人类基金 会”NGO(Non-Government Organization)于 2001 年 12 月在法国里尔市主办了第一届全球公约 大会，会议通过了《人类责任宪章》，并将构建“多元、尽责、协力”的世界作为重要的伦理 
目标。个体作为人类的未来，国家的希望，担负着维护世界和平发展，人类健康发展与自然 可持续发展的历史责任，只有将自我价值的实现与社会价值的实现相结合，在承担社会责任 的实践活动过程中才能真正体现出生命的价值。对社会责任品质的内涵进行国际比较, 既是 个体自身发展和社会发展的客观需求，也是现代教育发展的必然结果。

\section{2.社会责任品质内涵的国内研究}

社会责任品质在蜿蜒曲折的历史长河中屡经变迁，在主题和形态上不断发生着深刻的变 化; 然而, 在自身中对社会责任内涵的追求, 在思想中对社会责任境界的探寻, 在反思中对 社会责任实践的把握, 却是社会责任品质一以贯之、经久不衰的精华。国内关于社会责任品 质内涵的研究主要体现为以下三方面:

\section{1 伦理道德视角}

从伦理道德的视角出发, 学者认为社会责任品质是一种道德品质。“就个体来讲, 社会责 任品质是一种基础性的道德品质，提供了可能性；就社会来讲，社会责任是其良性发展的基 础, 尤其是在人际交往的广度及深度方面快速发展的现代经济社会”。[2]有学者认为作为社会 性个体间联结的内在基础, 责任具有丰富的道德内涵, 它既是一种社会规范, 更是个体道德 品质中较为核心的基础性品质, 与个体的其他品质相比, 责任具有基础性、内在性和选择性 特征。[3]作为一种基础性的道德品质, 社会责任品质的养成包括社会责任认知、社会责任情 感、社会责任意志和社会责任行为等心理过程。

\section{2 社会生活视角}

人的社会责任是社会规范对人的一种规定、一种使命，对具体的个体来说它是一种无可 推托、必须完成的“任务”。社会责任有两个方面的意义：一方面，人必须承担一定的职责和 任务, 这是由人的社会属性所决定的, 即积极承担自己所扮演社会角色的职责和任务。这既 是个体的生存手段和社会发展的必要条件，又是维系人与人之间、个人与社会、人与世界关 系的最基本的纽带。另一方面, 人必须对自身行为的后果负责。[4]落实科学发展观、建设和 谐社会等一系列宏大社会发展战略构想的达成, 最需要公众的道德精神, 最根本的要求就是 个体的社会责任品质。

\section{3 企业发展视角}

社会责任品质的内涵随着时代发展的步伐，一方面坚守着在历史长河的洗涤中保留下来 的优秀责任文化传统; 另一方面, 在全球化浪潮的包围下及社会转型期的巨变中, 在社会功 利主义、市场经济多元文化及自媒体等因素的影响下，面临新的挑战和风险。现实生活中， 公共产品肆意破坏、社会环境日益恶化、自然资源利用无度、社会秩序混沌失序等现象都折 射出了企业“社会责任品质”的堪忧。在我国全面深化改革的进程中，诸多社会风险和社会矛 盾都呼唤企业社会责任品质的彰显。企业的社会责任品质是企业通过保护环境、维护社会、 共享利益，从而达到和谐共赢，是企业发展的内在动力和精神品质。

\section{3.社会责任品质内涵的国外研究}

全球化的时代浪潮，使得各国之间文化融合、相互交流、相互影响的趋势逐渐增强。社 会责任品质的内涵研究也受到了国外学者的广泛关注。关于社会责任品质的内涵, 国外学者 从不同的角度出发，形成了以下三种具有代表性的观点。

\section{1 目标论}

国外学者将社会责任品质视为个体的基本道德品质, 是个体由片面走向整体, 最终实现 
全面发展的目标。社会责任品质是一种价值目标取向, 它植根于民主与他人的关系和道德原 则的关怀和正义。个体社会责任品质的发展应该成为个体发展的重点, 强调个体对社会责任 的践行, 积极关注他人, 并创造实践社会责任的机会。[5]社会责任品质是个体道德发展的需 要, 同时也是教育的培养目标。澳大利亚的高等教育机构一直在努力制定清晰的战略, 以便 在特定的学科背景下开发和评估毕业生的属性。将社会责任品质作为研究生的社会属性, 并 在评估任务中明确地嵌入需要考虑社会责任在实践经验中发展的情况, 将提升学生社会责任 品质这一目标的实现。 ${ }^{[6]}$ 此外, 人类社会发展的新变化, 也使得我们开始考虑将协同(a)社会 责任(SR)、(b)创新、(c)(辩证)系统理论, 作为实现必要的整体论 $(\mathrm{RH})$ 的理论。没有社会责任 $(\mathrm{SR})$, 所有人的利益都很难产生。没有社会责任(SR), 当前的文明也将几乎没有生存的机会。 社会责任 $(\mathrm{SR})$ 是一个人的属性。相互依存使人诚实，使人从片面走向整体。[7]

\section{2 要素论}

要素论者将社会责任品质作为系统中不可或缺的要素, 通过要素的完善来促进系统的优 化。Paolo Popoli 将企业社会责任作为企业社会创新的基本要素, 同时指出了企业社会责任对 企业社会的促进和保障功能。在超越企业社会责任的基础上，探讨链接企业社会责任(CSR) 和企业社会创新(CSI), 指出社会创新范式如何以及为什么通过克服 CSR 的传统“给予”逻辑来 代表商业机会, 从而使社会价值创造与经济价值创造携手并进。[8]也有学者将企业社会责任 作为一种国际战略，指出今天的公司负责公共项目的管理和执行，因此，企业是高度“社会责 任”的, 因为它们是代表公共利益行事的(尽管这不是他们做出的选择)。[9]迈克尔·波特和马 克·克莱默提出了一种全新的视角来看待商业和社会之间的关系, 这种关系并不把企业增长和 社会福利视为一种零和游戏而将社会责任看作是一种机会, 一种必不可少的要素。[10]社会责 任品质也是市场营销人员取得更大收益不可或缺的基本要素。[11]

\section{3 管理论}

管理论者将社会责任品质作为提升管理效能，解决社会、经济等领域危机的手段。《社 会和组织信息安全责任研究手册》， [12]揭示了当代社会管理和人力方面的信息安全问题，这 个具有里程碑意义的研究手册提供了对相关社会责任问题的评估与策略, 例如雇员监控、信 息安全策略和口令身份验证等。还有学者提出媒体的“社会责任论”, 主张新闻自由应受社会 制约，新闻传播要对社会负责的新闻理论，从而更好地实行“有控制的新闻自由”。[13]社会责 任品质的提升同时也为管理社会经济危机提供一种新的非技术创新。社会经济危机可以通过 社会责任 $(\mathrm{SR})$ 、人类必要的整体论 $(\mathrm{RH})$ 和幸福 $(\mathrm{WB})$ 来解决。SR 可以通过减少人的片面性来 帮助解决危机, 同时 SR 和 WB 都支持 RH 行为, 它们之间的创新协同作用导致了危机的解 决。[14]

\section{4. 结论}

通过社会责任品质内涵的国际比较，可以得出以下结论：社会责任品质是指在社会领域 由个体 (团体) 资格和角色资格所赋予的法律及道德品质。社会责任品质受生产力发展水平 及人的现代化发展水平所影响，不同国家，不同历史时期、不同个体的社会责任品质都有其 特殊性和独特性。社会责任品质的内涵可以从伦理和治理双重的维度进行全面考量。一方面, 社会责任品质作为个体道德品质的一部分具有重要的伦理意义; 另一方面, 个体作为社会关 系的总和，社会责任品质的优劣与国家治理现代化的水平息息相关。伦理与治理的双重内涵 既不是简单的平行关系, 也不是简单的相交关系，而是螺旋相交，形成合力的关系。未来对 社会责任品质的培育既要汲取历史的宝贵经验又要放眼未来; 既要立足于本国实际, 同时又 是人类文明的综合表达。理性认知社会责任品质的内涵, 对未来的研究能够提供有益的借鉴 和参考。 


\section{致谢}

基金项目: 全国高校辅导员研究基地江西师范大学招标课题; 江西省 2017 年青马工程项目; 江西师范大学 马克思主义学院 2017 年度青马课题（2017QMKT009）。

\section{References:}

[1] Xue Dezhen. Outline of Human Philosophy [M]. Beijing: People's Publishing House, 2005: 230.

[2] Song Ye. Moral Connotations of Responsibility and Mechanism of Realization [J]. Journal of Nanjing Normal University (Social Science Edition), 2003 (4): 89.

[3] Cui Xinwei. Outline of School Responsibility Education [M]. Beijing: China Social Sciences Press, 2012: 97-100.

[4] Cui Xinwei. Outline of School Responsibility Education [M]. Beijing: China Social Sciences Press, 2012: 141.

[5] Wray-Lake L, Syvertsen A K. The developmental roots of social responsibility in childhood and adolescence.[J]. New Directions for Child \& Adolescent Development, 2011, 2011(134):1125 .

[6] Howells, Kerry|Fitzallen, Noleine|Adams, Christine. Using Assessment to Develop Social Responsibility as a Graduate Attribute in Teacher Education.[J]. Australian Journal of Teacher Education, 2016, 41(6):52-67.

[7] Argandona A. Business Ethics and Corporate Social Responsibility[J]. Vital Speeches of the Day, 2008, :11(:1):216.

[8] Paolo Popoli.Social Enterprise and Social Innovation: A Look Beyond Corporate Social Responsibility[M].IntechOpen:2016-04-13.

[9] Christina Keinert.Corporate Social Responsibility as an International Strategy[M]. Physica-Verlag HD:2008-06-15.

[10] .Porter M E, Kramer M R. Strategy and society: the link between competitive advantage and corporate social responsibility[J]. Harvard Business Review, 2006, 84(12):78.

[11] Singhapakdi A, Rallapalli K C, Kraft K L, et al. The Perceived Role of Ethics and Social Responsibility: A Scale Development[M]// Proceedings of the 1994 Academy of Marketing Science (AMS) Annual Conference. Springer International Publishing, 2015:328-328.

[12] Gupta, Manish.Handbook of Research on Social and Organizational Liabilities in Information Security[M].IGI Global:2008-06-15.

[13] Hodges ， L.W.“Defining Press Responsibility : A Functional Approach."in D.Elliott(ed.).Responsible Journalism, Beverly Hills, CA : Sage, 1986, pp13-31.

[14] Žižek S Š, Mulej M. Social responsibility: a way of requisite holism of humans and their wellbeing[J]. Kybernetes, 2013, 42(1-2):318-335. 